\title{
Randomised trial of wide-field guided PRP for diabetic macular oedema treated with ranibizumab
}

\author{
S. James Talks ${ }^{1} \cdot$ Devangna Bhatia $^{1} \cdot$ Geeta Menon $^{2} \cdot$ Abosede Cole $^{3} \cdot$ Haralabos Eleftheriadis $^{4} \cdot$ Louise Downey $^{5}$. \\ Ngai Victor Chong $\mathbb{D}^{6} \cdot$ Sobha Sivaprasad $\mathbb{D}^{7} \cdot$ on behalf of the RDP study group
}

Received: 20 June 2018 / Revised: 12 November 2018 / Accepted: 22 December 2018 / Published online: 6 February 2019

(c) The Author(s) 2019. This article is published with open access

\begin{abstract}
Background Diabetic macular oedema (DMO) is effectively treated with ranibizumab but multiple injections are required. Where there is also peripheral ischaemia, it has been promoted that targeted panretinal photocoagulation (PRP) may reduce the number of injections.

Method Patients with optical coherence tomography confirmed DMO and Ultra-widefield Fundus Fluorescein Angiography confirmed peripheral retinal ischaemia were randomised to PRP plus ranibizumab or ranibizumab monotherapy. After three injections, repeat injections were given until the visual acuity was stable and the macula was dry. Re-treatment was given if there was a drop of visual acuity and/or a recurrence of intra-retinal fluid. The primary outcome was the number of repeat injections required after the first 6 months up until 1 year.

Results There were 49 patients, 25 in the ranibizumab only group and 24 in the ranibizumab + PRP group recruited at seven UK sites. The average number of injections in the ranibizumab-only arm was 6.84 over 1 year and 2.52 between months 6 and 12. The average number of injections in the combined arm was 6.67, with the number of injections in the second 6 months 1.92. For the primary outcome, comparing the number of 6- to 12-month injections, the result was not statistically significant $(p=0.33)$.

Conclusion The addition of targeted PRP to areas of non-perfusion in a patient with DMO does not reduce the number of injections required in the first year. It seems most likely that local VEGF at the macula is the main cause of DMO.
\end{abstract}

S. James Talks

James.talks@nuth.nhs.uk

1 Newcastle Eye Centre, Newcastle Upon Tyne NHS Hospitals Foundation Trust, Tyne and Wear, UK

2 Frimley Park Hospitals NHS Foundation Trust, Camberley GU16 7UJ, UK

3 University Hospitals Bristol NHS Foundation Trust, Bristol BS2 8AE, UK

4 King's College Hospital NHS Foundation Trust, London SE5 9RS, UK

5 Hull Royal Infirmary, Hull and East Yorkshire Hospitals NHS Trust, Hull HU3 2JZ, UK

6 Oxford Eye Hospital, Oxford University Hospitals NHS Foundation Trust, Oxford OX3 9DU, UK

7 Moorfields Eye Hospital NHS Foundation Trust, London EC1, UK

\section{Introduction}

Although centre-involving diabetic macular oedema (DMO) is effectively treated by anti-vascular endothelium growth factor (VEGF) injections, many patients require a large number of injections, at least in the first year. In the DRCR.net study (Protocol I) [1], a median of eight injections were required in the first year in the two ranibizumab arms. In the Restore study [2], a mean of seven injections were given in the first year. These treatments are expensive and cause a significant burden to the patient, their caregivers and the healthcare system.

The exact stimulus for VEGF production in DMO is unclear. In eyes with DMO and ischaemic peripheral retina [3], it has been postulated that the VEGF drive may be from the peripheral retina. Therefore, panretinal photocoagulation (PRP) targeted to peripheral non-perfused retina may reduce the VEGF load and in turn reduce the number of anti-VEGF injections required.

A recently published small trial, the DAVE study [4], did not find a reduction in the number of injections required 
over 3 years. This study evaluated 40 eyes of 29 patients and performed several sessions of PRP to ensure all the ischaemic areas of retina were treated. PRP itself can induce macular oedema and so possibly too much laser counteracted the potential benefit. Indeed, in this study there was less reduction of central retinal thickness (CRT) in the combined injection/PRP arm compared with monotherapy, $302 \mu \mathrm{m}$ compared with $152 \mu \mathrm{m}(p=0.03)$. The RDP study used a standard amount of laser targeted to the ischaemic area of peripheral retina, imaged by Ultra-widefield Fundus Fluorescein Angiography (UWFFA), as a one-off treatment to assess if the number of injections in the second 6 months of the first year of treatment was reduced. The second 6 months was pre-specified to make allowance for any possible initial worsening of the DMO due to the laser.

\section{Method}

The RDP study was a multicentre UK-based study run between 2014 and 2017. Patients with optical coherence tomography (OCT) (Heidelberg Engineering, Heidelberg, Germany) confirmed centre-involving DMO and UWFFA (Optomap P200, Optos PLC, Dunfermline, United Kingdom) confirmed peripheral retinal ischaemia were randomised to PRP plus ranibizumab or ranibizumab monotherapy.

Prior to any study-related procedures, all patients gave informed consent. The study had ethics approval (REC reference 13/NE/0197; IRAS 121940) and was registered with the clinical trial register (ISRCTN84503751). The study was performed in line with Good Clinical Practice in research guidelines and conformed with those of the Declaration of Helsinki.

The inclusion and exclusion criteria used are shown in Table 1. Eyes with new vessels were excluded. The assessment of the OCT scans and fundus fluorecein angiogram (FFA) was performed by the investigators to confirm eligibility. The images were subsequently sent to a reading centre.

Randomisation was by patients rather than eyes, as one eye per patient was included in the study. Which eye was chosen, if both were eligible, was at the investigator's

Table 1 Key inclusion and exclusion criteria

\begin{tabular}{|c|c|}
\hline Inclusion criteria & Exclusion criteria \\
\hline $\begin{array}{l}\text { Visual acuity } 20 / 32 \text { ( } 80 \text { EDTRS letters at } 4 \mathrm{~m} \text { ) to 20/320 (30 } \\
\text { EDTRS letters } 4 \mathrm{~m} \text { chart) }\end{array}$ & visual acuity worse than $20 / 320$ \\
\hline $\begin{array}{l}\text { Macular oedema secondary to diabetic retinopathy. (OCT } \\
\text { thickness of }>300 \mu \mathrm{m} \text { central subfield, on spectral domain OCT } \\
\text { (Spectralis Heidelberg engineering) }\end{array}$ & Rubeosis \\
\hline $\begin{array}{l}\text { Peripheral ischaemia seen on UWFFA ( } 20 \% \text { of the peripheral } \\
\text { retina or more) }\end{array}$ & Proliferative diabetic retinopathy \\
\hline \multirow[t]{12}{*}{ Patient able to give consent and take part in all study procedures } & $\begin{array}{l}\text { No recent change to antihypertensive treatment within } 2 \text { months of start of } \\
\text { study }\end{array}$ \\
\hline & $\begin{array}{l}\mathrm{BP}>180 / 110 \mathrm{mmHg} \text { (if elevated, treatment may be modified, and patients } \\
\text { may subsequently be considered for recruitment after at least } 2 \text { months on } \\
\text { new treatment regime) }\end{array}$ \\
\hline & Unable to give consent or take part in all study procedures \\
\hline & $\begin{array}{l}\text { Other conditions that might interfere with the assessment of the eye such as } \\
\text { cataract or prevent the macular oedema from settling such as vitreomacular } \\
\text { traction, epiretinal membrane, to a degree that would in the opinion of the } \\
\text { investigator affect response to treatment; conditions that would prevent the } \\
\text { visual acuity improving such as foveal atrophy, uveitis }\end{array}$ \\
\hline & Previous macular laser within 4 months in the study eye \\
\hline & Previous peripheral (PRP) laser in the study eye \\
\hline & Previous injection therapy within last 6 months in the study eye \\
\hline & Pregnant \\
\hline & $\begin{array}{l}\text { Uncontrolled systemic illness that in the opinion of the investigator would } \\
\text { preclude involvement }\end{array}$ \\
\hline & Systemic steroid treatment within 3 months of recruitment, or during the study \\
\hline & Cataract or other intraocular surgery within 3 months \\
\hline & Previous vitrectomy \\
\hline
\end{tabular}

ETDRS Early Treatment Diabetic Retinopathy Study, OCT optical coherence tomography, UWFFA Ultra-widefield Fundus Fluorescein Angiography, $B P$ blood pressure, $P R P$ panretinal photocoagulation 
discretion. Equal numbers of patients were randomised either to receive or not to receive PRP. Randomisation was performed via the randomisation service Sealed Envelope.

The ranibizumab treatment criteria were: three injections 28 days apart in all cases ( \pm 5 days) then repeat injections until two stable BCVA measures (within five LogMAR letters) and the macula was dry as judged by the investigator. Re-treatment was given if there was a drop of visual acuity of $>5$ letters, in the opinion of the investigator due to a recurrence of DMO, or a recurrence of intra-retinal fluid, as judged by the examining doctor. All patients were given three monthly ranibizumab injections and then followed up at monthly intervals with repeat visual acuity testing and OCT for a year. Repeat ranibizumab was given based on the specified re-treatment criteria.

No macular laser was planned but if the physician felt it was in the best interests of the patient to have some laser then it could have been applied but only after 6 months. No additional PRP was planned unless the patient developed rubeosis or new vessels that were not controlled by the injections.

\section{Intervention}

The intervention (PRP) arm was given 2000, 200 micrometre, laser spots with the Pascal laser (Topcon (GB), Newbury, United Kingdom), 2 weeks after the first ranibizumab injection, to the area of ischaemia seen on UWFFA. An UWF (Ultra-widefield) colour and UWFFA was repeated at 1 year.

\section{Comparator}

The comparator arm (ranibizumab monotherapy) did not receive any planned PRP.

\section{Assessments}

Best-corrected visual acuity (BCVA) was measured at $4 \mathrm{~m}$ on a standard Early Treatment Diabetic Retinopathy Study (ETDRS) chart at every visit. OCT was performed on the Heidelberg Spectralis OCT Machine looking at the CRT at every visit. UWFFA was captured on the Optomap P200, using eye steering to ensure the maximum area of retina was imaged.

\section{Outcome measures}

The primary outcome was the number of repeat ranibizumab injections required after the first 6 months up until 1year post treatment. Secondary outcomes were: the total number of injections given over 1 year; difference in BCVA at 1 year; changes in area of retinal ischaemia on UWFFA; changes in macular thickness (CRT) on OCT.

\section{Statistical analysis and sample size}

The statistical methods and sample size calculations to examine whether the number of repeat injections differed between the PRP and non-PRP groups was conducted based on intention to treat. In order to allow time for any effect of the PRP treatment to become evident, a lag period of 6 months was used; consequently, the number of injections during the final 6 months of the period of observation was analysed.

The average numbers of repeated injections in the PRP and non-PRP groups was compared on the basis that, for any patient the number of injections followed a binomial distribution with a possible maximum equal to the total number of monthly visits during the relevant period (i.e., six during the final 6 months of observation). To allow for the possibility that these numbers were not binomially distributed, a Mann-Whitney test was also used to test for differences between groups. If adjustment is required for other variables (e.g., level of ischaemia), then logistic regression was additionally performed.

In order to have $90 \%$ power to detect a difference between groups in the mean number of repeat injections during the final 6 months of the 12-month study period, based on a two-sided test at the 5\% level and assuming mean numbers 2.5 and 4 in the PRP and non-PRP groups, respectively, then a total of 28 patients would be required. Alternatively, if the mean numbers were 3 and 4, respectively, then a total of 60 patients would be required. Based on this, we opted for a target of 50 patients.

\section{Reading centre methodology for measuring retinal ischaemia}

The UWFFAs were read retrospectively. The images were sent to the Central Angiographic Resource Facility (CARF), Institute of Clinical Science, Queens University, Belfast and graded for degree of retinopathy and area of ischaemia at baseline and 1 year.

The method for measuring the area of ischaemia and change in ischaemia involved placing a designed grid centred on the foveal centre with the following zones: Foveal zone: diameter of $1.8 \mathrm{~mm}$ ( = 1DD); Macular zone: diameter of $7.2 \mathrm{~mm}$ (foveal circle is centralised within this area); Zone 1: diameter of $16.2 \mathrm{~mm}$; Zone 2: diameter of $25.2 \mathrm{~mm}$; Zone 3: diameter of $35.0 \mathrm{~mm}$. This was applied to the UWFFA images using automated software that calibrated the grid to the size of the image being graded, after the foveal centre was manually marked by the grader. The area beyond zone 3 was disregarded as it was found to be incomplete or ungradable in a majority of images (Fig. 1). Ischaemia was graded in the macular zone and zones 1-3. Each zone outside the macula was divided into four 


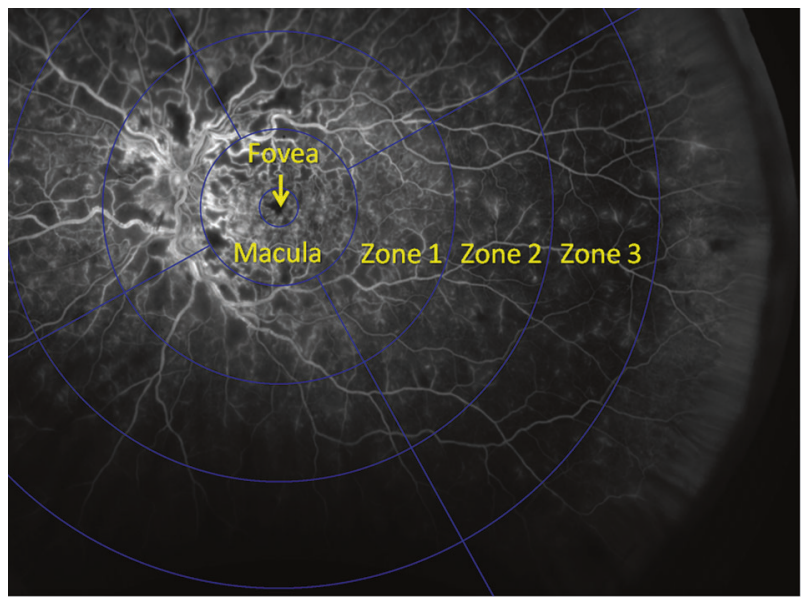

Fig. 1 Grid designed and placed on the foveal centre, to measure the area of and change in ischaemia

sectors-superior, inferior, temporal, and nasal as shown in Fig. 1. Within the macula and each of the 12 sectors, the total area of capillary non-perfusion (CNP) was estimated by an experienced grader and one of the following five grades assigned: no CNP, 1-25\%, 26-50\%, 51-75\%, 76$100 \%$. CNP was defined as an area of the capillary network that fails to fill with fluorescein by the late arteriovenous phase, with minimum linear dimension (MLD) $\geq 63 \mu \mathrm{m}$. If $>50 \%$ area of a sector was ungradable, then the grade 'Cannot grade' was assigned.

A final percentage CNP grade for total retinal nonperfusion was given for the gradable part of the retina by considering the individual grades for all the different zones graded. Areas with PRP laser could not be graded satisfactorily and therefore it was decided to not assign any scores to them. At year 1, images were graded in a similar manner and if new CNP was detected or an increase in CNP was found, this was stated and the retinal quadrant in which this was seen was asked to be specified.

\section{Results}

There were 49 patients recruited from 7 centres, 25 in the ranibizumab only group and 24 in the ranibizumab + PRP group. Eighty-seven percent completed 1-year follow-up. Three patients in the ranibizumab-only arm did not complete the study, one did not want to remain under follow-up and two died. Three did not complete in the combined arm, two did not want to remain under follow-up and one died. The groups were reasonably balanced with a mean baseline age of 63 years, $\mathrm{HbA} 1 \mathrm{c}$ of $80 \mathrm{mmol} / \mathrm{mol}$, blood pressure of $143 / 80 \mathrm{mmHg}$, OCT CRT of $391 \mu \mathrm{m}$, and area of ischaemia of $44 \%$. The mean baseline visual acuity was better in the ranibizumab-only arm at 73 ETDRS letters compared with 67 ETDRS letters (Table 2).
Both arms had two patients that had mild new vessels detected on the baseline FFA by the reading centre. These patients were also included in the analysis. The average number of injections in the ranibizumab-only arm was 6.84 and the number between 6 and 12 months was 2.52 (SD 2.24). The average number of injections in the combined arm was similar at 6.67, with the number of injections in the second 6 months being 1.92 (SD 2). For the primary outcome, comparing the number of 6- to 12-month injections, the result was not statistically significant $(p=0.33)$.

Similarly comparing the total number of injections in both groups, the differences were not statistically different $(p=0.84)$. The total number of injections also did not relate to the area of ischaemia in either arms or in the arms combined $(p=0.24)$.

There was a statistically significant improvement in visual acuity in each arm, with an average starting BCVA in the ranibizumab-only arm of 73.7 ETDRS letters improving to BCVA 77.8 letters ( +4 letters) and in the combined arm from BCVA 67.3 letters to BCVA 70.8 letters $(+3.5)$. Allowing for the difference in the baseline BCVA measurements, there was no difference in the mean change in the visual acuity between the arms although $84 \%(21 / 25)$ gained vision in the ranibizumab-only arm and only 16/24 $(66 \%)$ in the combined arm, using last observation carried forward for the three in each arm without final data. This difference was also not significant $(p=0.99)$. Two patients lost $>10$ letters in the ranibizumab arm only and none in the combined arm. One of these still had very good vision, having started at 95 letters, which dropped to 82. This may have been due to the development of some cataract. The macular oedema had dried up and the retinopathy grade of severe non proliferative diabetic retinopathy (NPDR) was the same at the end of the year. In the second case, the vision dropped from 75 letters to 56. The retinopathy grading remained as severe NPDR. The image quality at the end was worse consistent with cataract development, although there was persistent macular oedema. In the ranibizumab-only arm, 21/25 also had a final visual acuity of over 72 letters compared with 11/24 in the combined arm, although the starting vision was lower in this group.

There was a statistically significant improvement in the area of retinal ischaemia in the ranibizumab-only $\operatorname{arm}(p=$ $0.0045)$ compared with baseline but not in the combined arm $(p=0.29)$. Ten improved and one became more ischaemic in the ranibizumab arm despite 10 injections in the patient who became worse. In the combined arm, seven improved and four became more ischaemic, the rest having the same area of ischaemia (Table 3; Figs. 2a, b).

The OCT thickness decreased by a similar amount in both groups - in the ranibizumab-only arm from $378 \mu \mathrm{m}$ to $318 \mu \mathrm{m}$ and in the combined from $405 \mu \mathrm{m}$ to $310 \mu \mathrm{m}$ (Fig. 3). 
Table 2 Patient demographics

\begin{tabular}{|c|c|c|c|c|}
\hline & Ranibizumab only & Ranibizumab+PRP & Total & $p$-Values \\
\hline No. of eyes & 25 & 24 & 49 & N/A \\
\hline No. of patients & 25 & 24 & 49 & N/A \\
\hline Right eye \% (no/total) & $64 \%(19 / 25)$ & $50 \%(12 / 24)$ & $63 \%(31 / 49)$ & N/A \\
\hline Age (years), mean (range) & $62.64(40-84)$ & $64.91(48-83)$ & $63.73(40-84)$ & 0.4082 \\
\hline Women \% (no/total) & $16 \%(4 / 25)$ & $25 \%(6 / 24)$ & $20.41 \%(10 / 49)$ & N/A \\
\hline HbA1c mean (range) & $77.78(45-113)$ & $82.20(55-111)$ & $80.11(45-113)$ & 0.6464 \\
\hline BP (mmHg) (average) & $138 / 80$ & $148 / 81$ & $143 / 80$ & $\begin{array}{l}0.1553 / \\
0.7778\end{array}$ \\
\hline PDR \% (no/total) & $8 \%(2 / 25)$ & $8.33 \%(2 / 24)$ & $8.16 \%(4 / 49)$ & N/A \\
\hline $\begin{array}{l}\text { Beginning BCVA mean } \\
\text { (range) }\end{array}$ & $73.68(48-95)$ & $67.29(52-89)$ & $70.55(48-95)$ & 0.0229 \\
\hline Ending BCVA mean (range) & $77.88(56-92)$ & $70.79(52-89)$ & $74.41(52-92)$ & 0.0177 \\
\hline $\begin{array}{l}\text { Beginning CRT }(\mu \mathrm{m}) \text { mean, } \\
\text { (range) }\end{array}$ & $378.36(308-532)$ & 405.67 (301-1023) & $\begin{array}{l}391.74(301- \\
1023)\end{array}$ & 0.4704 \\
\hline $\begin{array}{l}\text { Ending CRT }(\mu \mathrm{m}) \text { mean, } \\
\text { (range) }\end{array}$ & $316(224-427)$ & $310.79(180-541)$ & $\begin{array}{l}313.45(180- \\
541)\end{array}$ & 0.7346 \\
\hline $\begin{array}{l}\text { Beginning ischaemia (\%) } \\
\text { mean, (range) }\end{array}$ & $43 \%(25-75 \%)$ & $45.45 \%(0-100 \%)$ & $\begin{array}{l}44.15 \%(0- \\
100 \%)\end{array}$ & 0.8621 \\
\hline $\begin{array}{l}\text { Ending ischaemia (\%) mean, } \\
\text { (range) }\end{array}$ & $29.55 \%(0-50 \%)$ & $39.47 \%(0-75 \%)$ & $34.15 \%(0-75 \%)$ & 0.2301 \\
\hline
\end{tabular}

$B C V A$ best-corrected visual acuity, $B P$ blood pressure, $C R T$ central retinal subfield thickness,

ETDRS Early Treatment Diabetic Retinopathy Study, HbAlc glycated haemoglobin, PDR proliferative diabetic retinopathy

Table 3 Comparing the grade of retinopathy and proportion of ischaemia in both groups

\begin{tabular}{llllll}
\hline & \multicolumn{2}{l}{ Ranibizumab $(n=25)$} & \multicolumn{2}{l}{$\begin{array}{l}\text { Ranibizumab }+ \text { PRP } \\
(n=24)\end{array}$} \\
\cline { 2 - 3 } \cline { 5 - 6 } & $\begin{array}{l}\text { Grade of } \\
\text { retinopathy }\end{array}$ & $\begin{array}{l}\text { Proportion } \\
\text { of ischaemia }\end{array}$ & $\begin{array}{l}\text { Grade of } \\
\text { retinopathy }\end{array}$ & $\begin{array}{l}\text { Proportion } \\
\text { of ischaemia }\end{array}$ \\
\hline No change & 18 & 11 & 16 & 9 \\
Improvement & 3 & 10 & 3 & 7 \\
Worsening & 4 & 1 & 4 & 4 \\
Unknown & 0 & 3 & 1 & 4 \\
\hline
\end{tabular}

No significant difference was found in either group when comparing the following: OCT thickness at baseline and the area of ischaemia (ranibizumab arm $p=0.72$, combined arm $p=0.17$ ); reduction of OCT thickness at year 1 and the area of ischaemia (ranibizumab arm $p=0.15$, combined $\operatorname{arm} p=$ 0.32 ); total number of injections and initial ischaemia (ranibizumab arm $p=0.65$, combined arm $p=0.28$ ); total number of injections and improvement in ischaemia (ranibizumab arm $p=0.79$, combined arm $p=0.98$ ).

Looking at the OCT thickness at baseline of all the patients $(n=49)$, there was no statistically significant difference when compared with the initial area of retinal ischaemia $(p=0.17)$. The speed of reduction of the OCT thickness did not vary between the two arms (Fig. 3).
In the ranibizumab arm, FFA at 1 year found an additional two patients had developed new vessels and an additional three patients in the combined arm. No additional laser had been required during the study. No patients had any serious ocular adverse events, in particular no endophthalmitis, although two patients lost $>10$ letters of vision. Nineteen serious adverse events (SAEs) were reported, requiring hospital admission, evenly distributed between the two groups, including three deaths. The deaths were related to heart disease and the other SAEs were largely related to complications of diabetes including kidney disease.

\section{Discussion}

Trials using anti-VEGF therapy show that a high number of treatments are required $[1,2]$. In the DRCR.net study Protocol I [1], only a total of 17 eyes (9\%) in the ranibizumab + prompt laser group and 15 eyes $(8 \%)$ in the ranibizumab + deferred laser group met 'success' criteria at 16 weeks and did not receive an additional injection before the 1-year primary outcome visit. In the RDP study, the addition of peripheral laser did not reduce the number of injections in total or in the second 6 months. More patients gained vision in the ranibizumab-only arm although a significant difference in the mean vision change was not observed. The mean 
Fig. 2 a Ranibizumab and laser arm: white arrows showing the areas of change and re-perfusion in the month 12 image compared with baseline. (Images chosen at the closest time point in FFA run). b Ranibizumab arm: white arrows showing the areas of change and re-perfusion in the month 12 image compared with baseline. (Images chosen at the closest time point in FFA run)
Fig. 3 Changes in optical coherence tomography (OCT) thickness from baseline in both the study arms

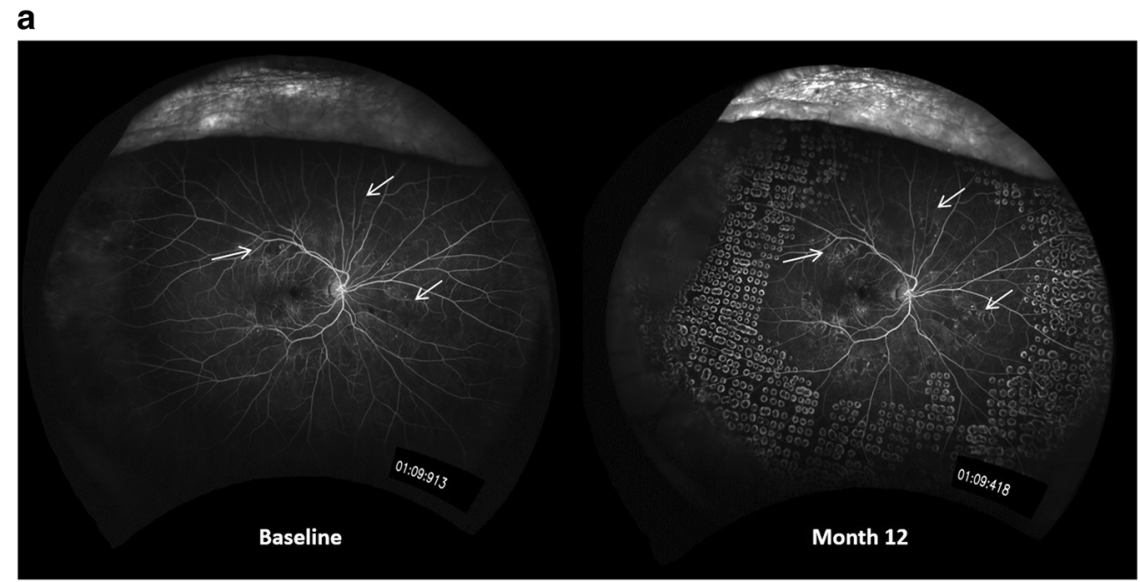

b

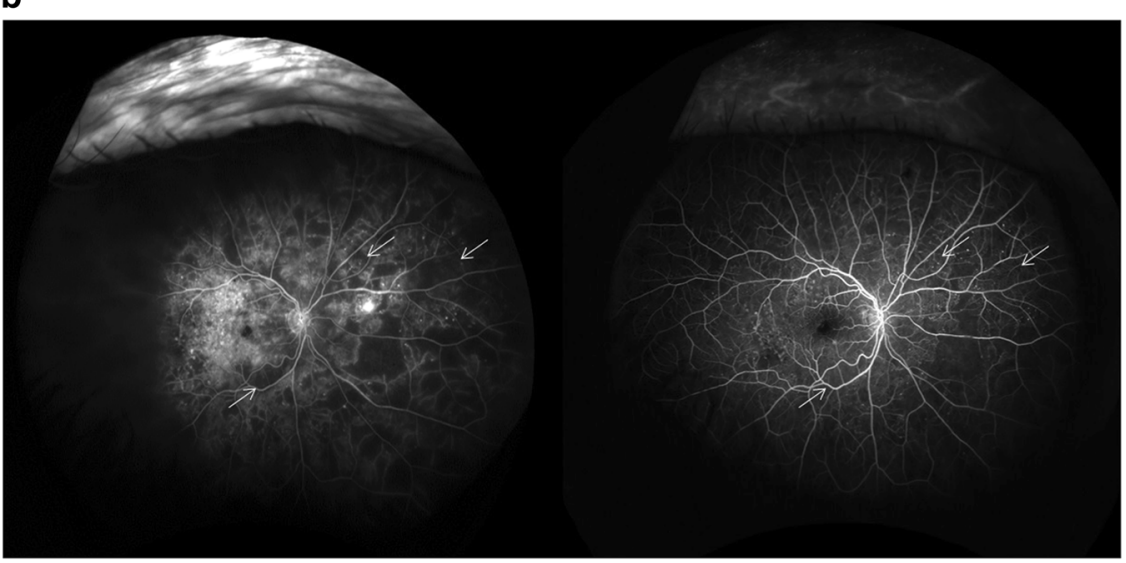

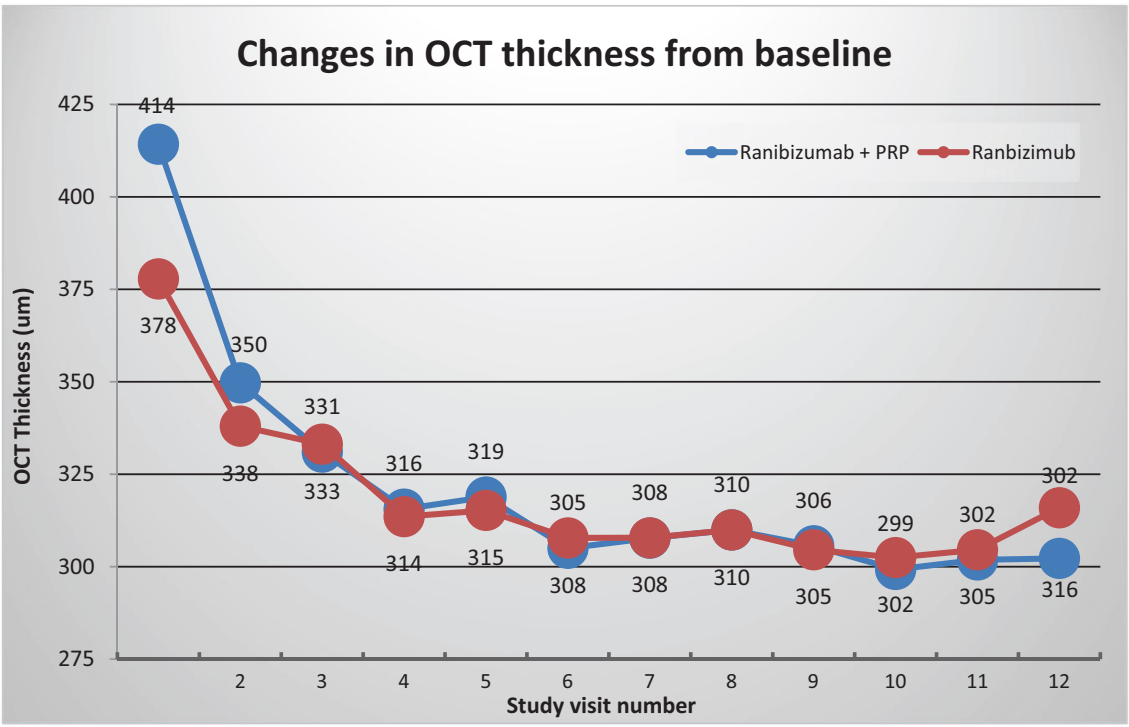

number of ETDRS letters gained in either arm was less than expected compared with other studies, however, the starting vision was higher and the OCT thickness lower. In the DAVE study [4], there was also a suggestion that the vision improvement was not as good in the combined arm. The number of injections given in the RDP was a little lower than might have been expected in both arms, at 6.7 compared with the median number of 8 injections in the two arms of the DRCR.net Protocol I study, although similar to the mean number of 7 used in the Restore study.

There was some improvement in the overall area of retina measured as perfused in the ranibizumab arm but not 
in the combined arm. This may not be a fair comparison as the area of retina measured varied as it was not possible to make a clear judgement of perfusion in the areas lasered. Judging changes in areas of perfusion can also be difficult. It maybe that optocal coherence tomography angiography (OCTA) would be better for this but this was not done and currently technology limits the retinal field that can be imaged with OCTA. Neither ranibizumab monotherapy nor the addition of laser prevented the development of new vessels completely. The hypothesis was that treating the non-perfused area of the peripheral retina would reduce the amount of VEGF and so lead to reduced anti-VEGF therapy. Ultra widefield FFA (UWF-FFA) has been shown to better demonstrate the full extent of diabetic retinopathy compared with seven-field imaging [5] but the association of peripheral retinal non-perfusion with diabetic maculopathy is not clear. Sim et al. [6] found an association of peripheral retinal ischaemia with an enlarged foveal avascular zone but not macular oedema, whereas a study by Wessel showed a correlation [3].

In a study of 148 eyes from 76 patients, before the advent of anti-VEGF injections, using UWF-FFA to measure the area of non-perfusion, eyes with larger areas of retinal nonperfusion and worse diabetic retinopathy needed more macular laser [7].

In a study of 99 eyes being treated for DMO with ranibizumab, $49 \%$ were associated with microaneurysms, $37 \%$ with peripheral ischaemia and $15 \%$ with neovascularisation. The oedema associated with peripheral ischaemia was more likely to have a diffuse as opposed to focal pattern and there was a positive correlation of response to the ischaemic index. Whether treating this ischaemia would reduce the recurrence rate was not addressed [8].

Two trials combining UWFFA targeted laser with antiVEGF treatments suggested that this combination was effective, however, they only had 6 months follow-up and other variables make the conclusions uncertain $[9,10]$. The RaScaL trial [10] studied 30 eyes of 22 patients randomised to a combination therapy of ranibizumab plus UWFFAguided laser compared with a control group that received combination therapy of intravitreal triamcinolone plus focal macular laser. Fewer patients in the ranibizumab plus laser arm needed re-treatment. Takamura et al. [9] randomised 52 patients to bevacizumab or bevacizumab plus targeted PRP then gave all patients focal laser 2 weeks later and monitored the increase in CRT. In the group with the additional targeted PRP they found less increase, and this also related to the area of non-perfusion. Two-thirds of eyes already had already received standard PRP at baseline.

The Relate study [11] and a small study by Spaide [12] found that the addition of PRP to ranibizumab injections for macular oedema due to central retinal vein occlusion did not show a benefit.
In an analysis of the baseline images of the DAVE study [13] involving 40 eyes of 29 patients, randomised to antiVEGF with or without laser to peripheral ischaemia, the nonperfused area and total retinal area visible was used to calculate an ischaemic index in different retinal zones and correlated to the severity of DMO. The ischaemic index increased with increasing distance from the fovea but the severity of the oedema did not correlate with the overall nonperfused area or ischaemic index [13]. A measure of overall leakage may better relate to VEGF levels but quantifying leak from FFA photographs is difficult as the brightness of an image varies along the run depending on the light exposure.

In the RDP study, one PRP laser of 2000 shots, using the PASCAL laser, was applied to the ischaemic area found on UWF-FFA. This amount was chosen as it was felt it would give some consistency between investigators, would not be too small an amount to have an effect but would be unlikely to risk visual field loss for what was an unproven treatment. This might have limited the benefit of the laser, as the amount could have been varied depending on the amount of ischaemia, however, in the DAVE study [4], heavier titrated laser was applied and repeated during the course of the study. Both approaches did not show an effect. Both studies are small but do not suggest that the addition of laser to ranibizumab monotherapy is beneficial or more precisely the addition of PRP is not more efficient at reducing VEGF levels than antiVEGF alone. Laser may in fact reduce the visual acuity gains that is achieved with ranibizumab monotherapy. This might be because PRP does not reduce the VEGF levels enough in the vitreous to affect the macula and that the non-perfused area on the FFA is not the only source of cytokines, which promote the development of macular oedema.

Putting the two studies together, it seems most likely that posteriorly produced VEGF or local intra-retinal VEGF and other cytokines at the macula are the main cause of macular oedema.

\section{Summary}

\section{What was known before}

- Ranibzumab works well for diabetic macular oedema (DMO) but many injections are needed in the first year.

- Ischaemic retina produces VEGF and blocking VEGF reduces macular oedema.

- PRP can reduce VEGF levels.

\section{What this study adds}

- The addition of PRP to ranibizumab treatment for DMO does not reduce the number of injections required in the first year of ranibizumab therapy. 
Acknowledgements We acknowledge the support of the research teams in each unit and the work of the image reading centre. Central Angiographic Resource Facility (CARF), Institute of Clinical Science, Queens University, Belfast.

The RDP study group James Talks, Newcastle Eye Centre, Newcastle Upon Tyne NHS Hospitals Foundation Trust Geeta Menon, Frimley Park Hospitals NHS Foundation Trust; Abosede Cole, University Hospitals Bristol NHS Foundation Trust; Sobha Sivaprasad, Moorfields Eye Hospital NHS Foundation Trust; Ngai Victor Chong, Oxford Eye Hospital, Oxford University Hospitals NHS Foundation Trust; Haralabos Eleftheriadis, King's College Hospital NHS Foundation Trust; Louise Downey, Hull Royal Infirmary, Hull and East Yorkshire Hospitals NHS Trust.

Funding This study received funding and ranibizumab drug product support from Novartis Pharmaceuticals UK Ltd. Novartis had no role in the study design, collection, analysis, or interpretation of the data or decisions regarding this study.

\section{Compliance with ethical standards}

Conflict of interest Investigator-led trial. SJT—advisory board work for Novartis, Bayer; Optos, has received travel grants from Novartis, Bayer and Allergan; Honoraria for presentations from Novartis, Bayer. DBinvolved in research from Bayer and Novartis. GM-has received research and travel grants from Novartis and Bayer. AC-has received educational travel grants from Novartis \& Bayer. SS - has received travel fees, honorarium for advisory board and research grants from Bayer, Allergan, Novartis and Roche, Boehringer Ingleheim. SS has received honorarium for advisory board from Optos Plc and Heidelberg Engineering. NVC - at the time of the trial he worked as an ophthalmologist at the Oxford Eye Hospital, now he is an employee of Boehringer Ingelheim International $\mathrm{GmBH}$. HE-received travelling grants, honoraria from Novartis and participated in Board meetings. LD-advisory board work for Novartis, Bayer, Alimera and Allerga; has received travel grants from Novartis, Bayer and Allergan; Honoraria for presentations from Novartis, Bayer, Alimera.

Publisher's note: Springer Nature remains neutral with regard to jurisdictional claims in published maps and institutional affiliations.

Open Access This article is licensed under a Creative Commons Attribution 4.0 International License, which permits use, sharing, adaptation, distribution and reproduction in any medium or format, as long as you give appropriate credit to the original author(s) and the source, provide a link to the Creative Commons license, and indicate if changes were made. The images or other third party material in this article are included in the article's Creative Commons license, unless indicated otherwise in a credit line to the material. If material is not included in the article's Creative Commons license and your intended use is not permitted by statutory regulation or exceeds the permitted use, you will need to obtain permission directly from the copyright holder. To view a copy of this license, visit http://creativecommons. org/licenses/by/4.0/.

\section{References}

1. Diabetic Retinopathy Clinical Research Network, Elam MJ, Aiello LP, Beck RW, Bressler NM, Bressler SB, et al. Randomized trial evaluating ranibizumab plus prompt or deferred laser or triamcinolone plus prompt laser for diabetic macular edema. Ophthalmology. 2010;117:1064-77.

2. Mitchell P, Bandello F, Schmidt-Erfurth U, Lang GE, Massin P, Schlingemann RO, et al. The RESTORE study: ranibizumab monotherapy or combined with laser versus laser monotherapy for diabetic macular edema. Ophthalmology. 2011;118:615-25.

3. Wessel MM, Nair N, Aaker GD, Ehrlich JR, D'Amico DJ, Kiss S. Peripheral retinal ischaemia, as evaluated by ultra-widefield fluorescein angiography, is associated with diabetic macular oedema. Br J Ophthalmol. 2012;96:694-8.

4. Brown DM, Ou WC, Wong TP, Kim RY, Croft DE, Wykoff CC, et al. Targeted retinal photocoagulation for diabetic macular edema with peripheral retinal nonperfusion: three-year randomized DAVE trial. Ophthalmology. 2017;125:683-90.

5. Talks SJ, Manjunath V, Steel DHW, Peto T, Taylor R. New vessels detected on wide-field imaging compared to two-field and seven-field imaging: implications for diabetic retinopathy screening image analysis. Br J Ophthalmol. 2015;12:1606-9.

6. Sim DA, Keane PA, Rajendram R, Karampelas M, Selvam S, Powner MB, et al. Patterns of peripheral retinal and central macula ischemia in diabetic retinopathy as evaluated by ultra-widefield fluorescein angiography. Am J Ophthalmol. 2014;158:144-53.

7. Patel AJ, Downes K, Davis A, Das A. Are proliferative diabetic retinopathy and diabetic macular edema two different disease processes? A retrospective cross-sectional ctudy. Invest Ophthalmol Vis Sci. 2012;53:377.

8. Xue K, Yang E, Chong NV. Classification of diabetic macular oedema using ultra-widefield angiography and implications for response to anti-VEGF therapy. $\mathrm{Br} \mathrm{J}$ Ophthalmol. 2017; 101:559-63.

9. Takamura Y, Tomomatsu T, Matsumura T, Arimura S, Gozawa M, Takihara, et al. The effect of photocoagulation in ischemic areas to prevent recurrence of diabetic macular edema after intravitreal bevacizumab injection. Investig Opthalmology Vis Sci. 2014; 55:4741.

10. Suñer IJ, Peden MC, Hammer ME, Grizzard WS, Traynom J, Cousins SW. RaScaL: a pilot study to assess the efficacy, durability, and safety of a single intervention with ranibizumab plus peripheral laser for diabetic macular edema associated with peripheral nonperfusion on ultrawide-field fluorescein angiography. Ophthalmologica. 2014;233:89-95.

11. Campochiaro PA, Hafiz G, Mir TA, Scott AW, Solomon S, Zimmer-Galler, et al. Scatter photocoagulation does not reduce macular edema or treatment burden in patients with retinal vein occlusion: the RELATE trial. Ophthalmology. 2015;122:1426-37.

12. Spaide RF. Prospective study of peripheral panretinal photocoagulation of areas of nonperfusion in central retinal vein occlusion. Retina. 2013;33:56-62.

13. Fan W, Wang K, Ghasemi Falavarjani K, Sagong M, Uji A, Ip M, et al. Distribution of nonperfusion area on ultra-widefield fluorescein angiography in eyes with diabetic macular edema: DAVE study. Am J Ophthalmol. 2017;180:110-6. 